\title{
Recent Progress in Vector Vortex Coronagraphy
}

\author{
E. Serabyn*a , D. Mawet ${ }^{\mathrm{b}}$, J.K. Wallace ${ }^{\mathrm{a}}$, K. Liewer ${ }^{\mathrm{a}}$, J. Trauger ${ }^{\mathrm{a}}$, D. Moody ${ }^{\mathrm{a}}$, and B. Kern ${ }^{\mathrm{a}}$ \\ ${ }^{a}$ Jet Propulsion Laboratory, California Institute of Technology, Pasadena, CA, USA 91109 \\ ${ }^{b}$ European Southern Observatory, Alonso de Cordova 3107, Vitacura 7630355, Santiago, Chile
}

\begin{abstract}
The optical vortex coronagraph has great potential for enabling high-contrast observations very close to bright stars, and thus for reducing the size of space telescopes needed for exoplanet characterization missions. Here we discuss several recent developments in optical vortex coronagraphy. In particular, we describe multi-stage vortex configurations that allow the use of on-axis telescopes for high-contrast coronagraphy, and also enable the direct measurement of the amplitudes and phases of focal plane speckles. We also briefly describe recent laboratory demonstrations of the optical properties of the dual-stage vortex, and of the broadband performance of single stage vortex masks. Indeed, the demonstrated performance of the vector vortex phase masks already in hand, $\approx 10^{-8}$, is approximately that needed for an initial coronagraphic mission, such as an exoplanet explorer, aimed at detecting exozodiacal light and jovian exoplanets.
\end{abstract}

Keywords: vortex, coronagraphy, exoplanets

\section{INTRODUCTION}

An ideal coronagraphic space mission for the imaging of nearby solar systems would be both inexpensive and technically simple, and the optical vortex coronagraph ${ }^{1}$ has a number of favorable attributes in this regard, especially as it can share a common configuration with a classical Lyot coronagraph. Table 1 summarizes a range of desirable properties for a high-contrast coronagraphic system, and the potential solutions that an optical vortex based system can provide. The primary reasons for considering an optical vortex based approach are of course the small inner working angle and large, unobstructed field of view that this approach provides.

Table 1. Desirable capabilities of a space coronagraph and potential solutions provided by vortex phase masks.

\begin{tabular}{|c|c|c|}
\hline Subsystem/metric & Goal & Solution in Vortex/Phase Case \\
\hline Telescope & Small & Phase masks in general \\
\hline Telescope & On-axis optics & Multi-stage vortex coronagraph \\
\hline Flux throughput & High throughput for exoplanet light & Phase-based vortex mask \\
\hline Flux throughput & Broadband operation & $\begin{array}{l}\text { Multi-layer vortex or } \\
\text { Spectral polarization filtering }\end{array}$ \\
\hline Flux throughput & Dual-polarization operation & $\begin{array}{l}\text { Multi-layer vortex or } \\
\text { Spectral polarization filtering }\end{array}$ \\
\hline Field of view & Small inner working angle & Phase masks in general \\
\hline Field of view & Large outer working angle & Phase masks in general \\
\hline Field of view & All azimuths accessible & Vortex phase mask \\
\hline Wavefront quality & $\begin{array}{l}\text { Low sensitivity to low-order wavefront errors } \\
\text { (e.g., pointing, focus...), or ability to correct them }\end{array}$ & Higher order vortex phase mask \\
\hline Wavefront quality & Ability to sense and correct wavefront errors & $\begin{array}{l}\text { Direct speckle amplitude } \& \text { phase } \\
\text { sensing in a multi-stage vortex }\end{array}$ \\
\hline
\end{tabular}

UV/Optical/IR Space Telescopes and Instruments: Innovative Technologies and Concepts V, edited by Howard A. MacEwen, James B. Breckinridge, Proc. of SPIE Vol. 8146, 81460L · @ 2011 SPIE CCC code: $0277-786 X / 11 / \$ 18 \cdot$ doi: $10.1117 / 12.895267$ 
The final column of the table shows that an optical vortex coronagraph provides potential solutions in all areas listed. Most importantly, phase masks in general allow smaller telescopes to be considered. However, one recent development is that a multi-stage vortex configuration allows for the possibility of an on-axis telescope ${ }^{2}$. Moreover, the dual-stage vortex approach also allows for the direct measurement of speckle phases ${ }^{3}$, and thus can provide for robust wavefront sensing. In addition, vector vortex phase masks enable unique approaches to the broadband performance issue ${ }^{4}$. However, to be fair, it must be pointed out that not every one of the individual solutions are proven compatible with each other in a single configuration. Several of the aspects listed in Table 1 have been discussed before, thus here we focus on advantages of the multi-stage configuration, and on recent laboratory measurements showing broadband performance.

\section{SINGLE- AND DUAL-STAGE VORTEX CONFIGURATIONS: THEORY}

For a clear circular aperture, modification of the focal plane Airy pattern by passage through a single vortex leads to all of the light from an on-axis source being moved to the exterior of the image of the input pupil, as shown in Figure 1. This situation applies to the case of a clear off-axis telescope.

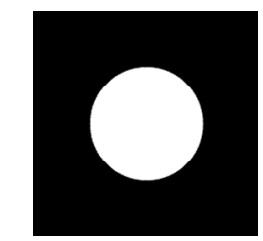

Input

pupil

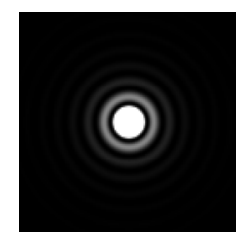

Focal

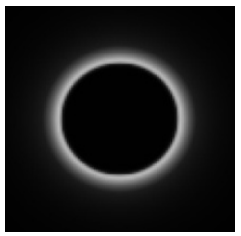

Pupil

plane
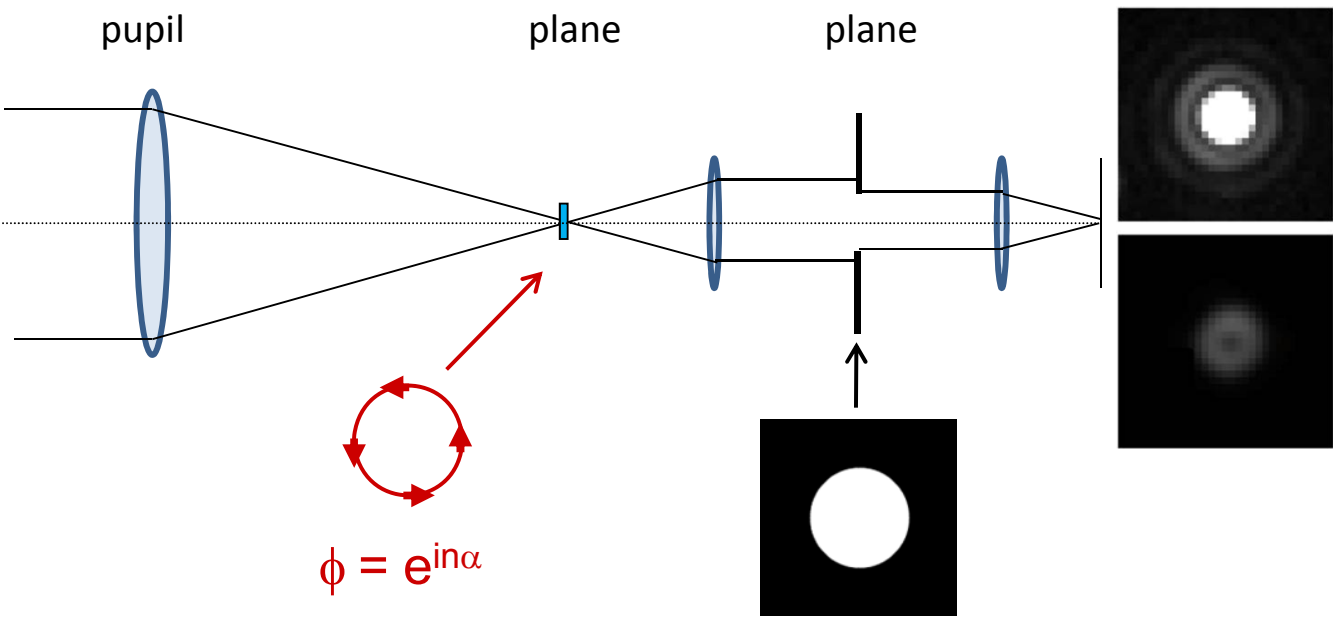

bypass vortex

through

vortex

Figure 1. Operation of a single-stage vortex coronagraph with a clear aperture.

On the other hand, in a two-mirror on-axis telescope configuration, the secondary mirror introduces extra diffraction into the pupil, as depicted in Fig. 2 (third image from the top). However ${ }^{2}$, if the light exterior to the input pupil is blocked in the first Lyot plane (fourth image), and the remaining light is passed through a second vortex, most of the residual light will appear (sixth image) as a uniform plane wave, at the original intensity, in the region originally blocked by the secondary. (A similar effect is seen in multi-stage four-quadrant phase mask coronagraphs ${ }^{5}$ ). This residual central light can then be blocked by a second Lyot stop in the second pupil/Lyot plane (bottom image in Fig. 2), thus leaving a replica of the original pupil, with only $(\mathrm{d} / \mathrm{D})^{4}$ of the starlight remaining in the outer region of the pupil, where $\mathrm{d}$ and $\mathrm{D}$ are the diameters of the secondary and primary mirrors, respectively. With a $10 \%$ linear blockage, this leaves only $10^{-4}$ of the stellar light in the pupil, which will be focused as usual, into an Airy pattern, with $\sim 10^{-7}$ of the original central brightness in the third Airy ring. A four-stage vortex configuration would square this effect, leaving only (d/D) ${ }^{8}$ or $10^{-8}$ of the light in the pupil, with $10^{-11}$ at the third Airy ring. 


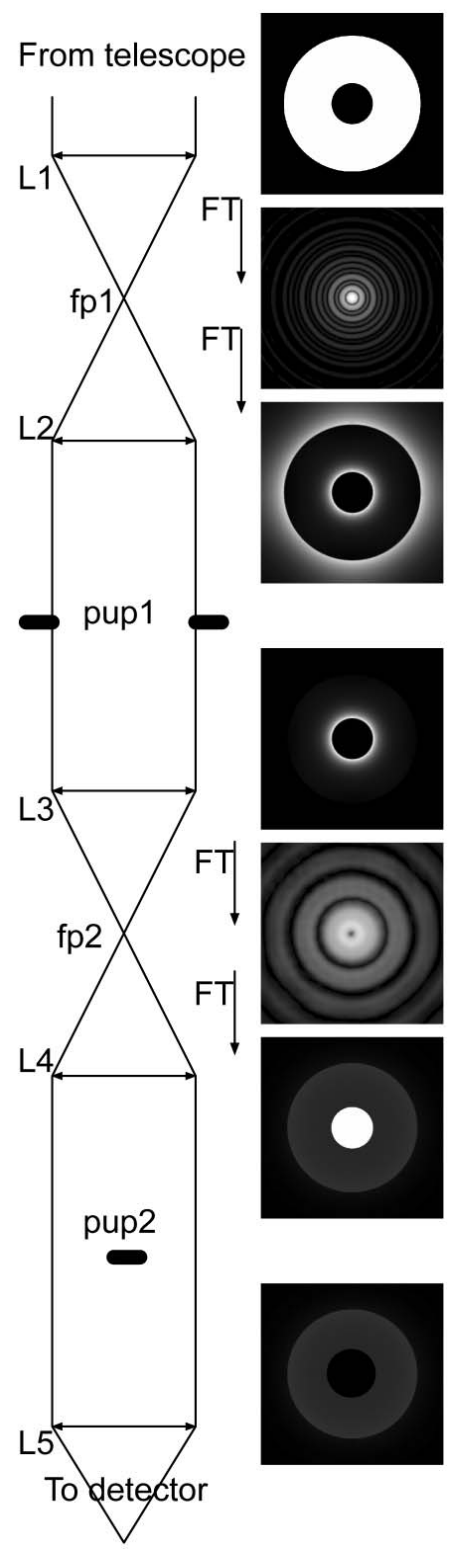

Figure 2. Operation of a dual-stage tandem vortex coronagraph ${ }^{2}$. See text for explanation.

\section{SINGLE- AND DUAL-STAGE VORTEX CONFIGURATIONS: MEASUREMENTS}

In order to test the dual-stage vortex concept, we have recently modified the Infrared Coronagraphic Testbed (IRCT) at JPL (Fig. 3) to accommodate both single- and dual-stage vortex coronagraph configurations. Figs. 4 through 6 show the pupil measurements obtained on the IRCT in both configurations, using a pair of second order vortex masks manufactured by Beam Co.

Fig. 4 compares the measured input and output pupils for a clear input aperture and a single vortex. The output pupil shows a dark center and a radial brightness falloff outside of the pupil, as expected. Next, Fig. 5 shows the input and output pupils for a centrally-blocked input aperture and a single vortex, and the output pupil shows the expected extra leakage around the central blockage. Finally, Figure 6 shows the first and second Lyot plane distributions in a double vortex configuration, for a centrally-blocked input aperture, after apodizing the first Lyot plane by an iris diaphragm (compare Fig. 5, right, with Fig. 6, left). The light distributions measured for the first and second pupil planes are indeed 
as predicted in Fig. 2: the light distribution in the second Lyot plane is dominated by a bright central component filling the region corresponding to the original central blockage. This bright central starlight beam can then be blocked in the second pupil plane with an additional Lyot stop.

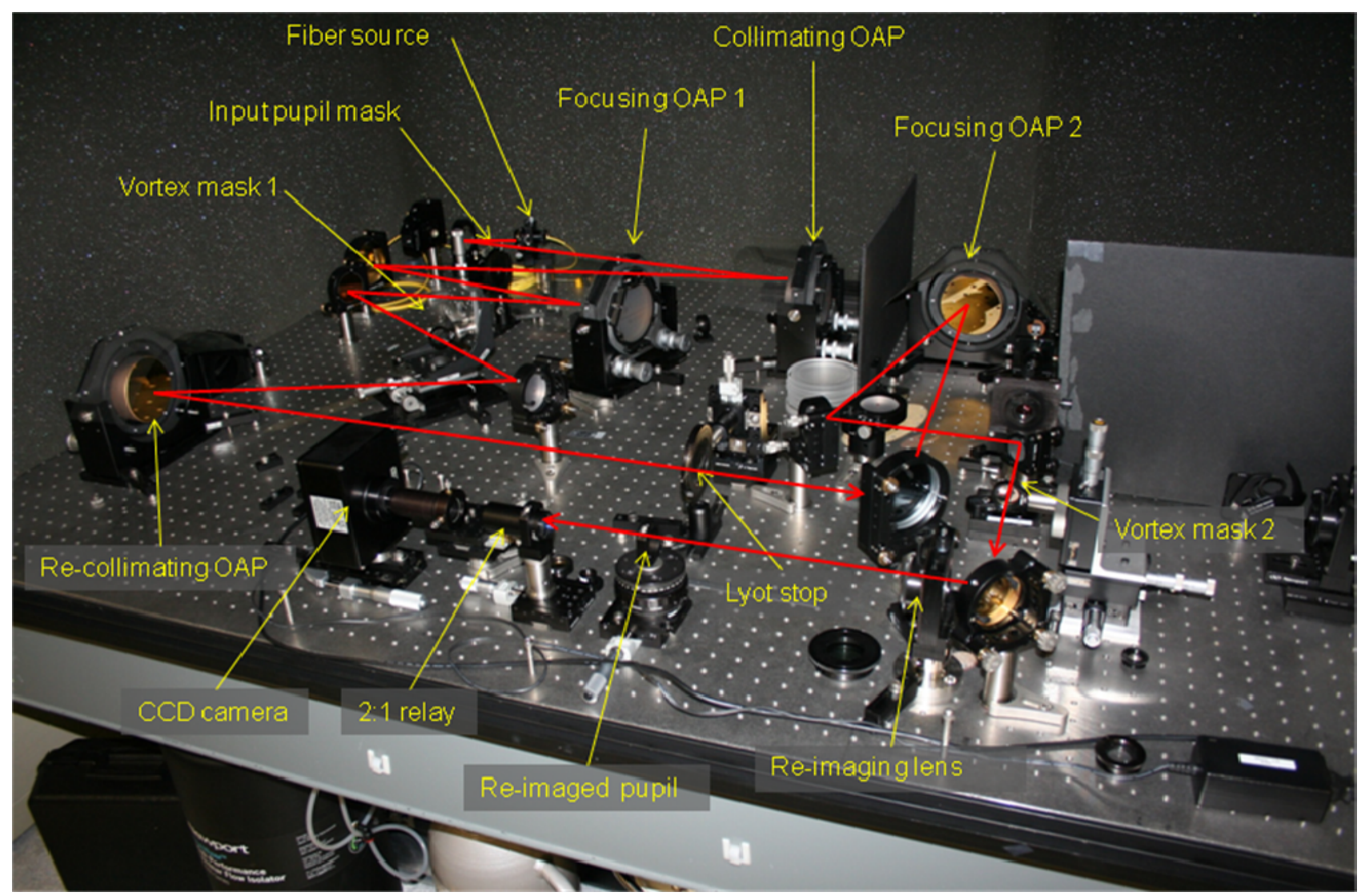

Figure 3. The Infrared Coronagraphic Testbed (IRCT) at JPL, with the dual-vortex beam path highlighted.
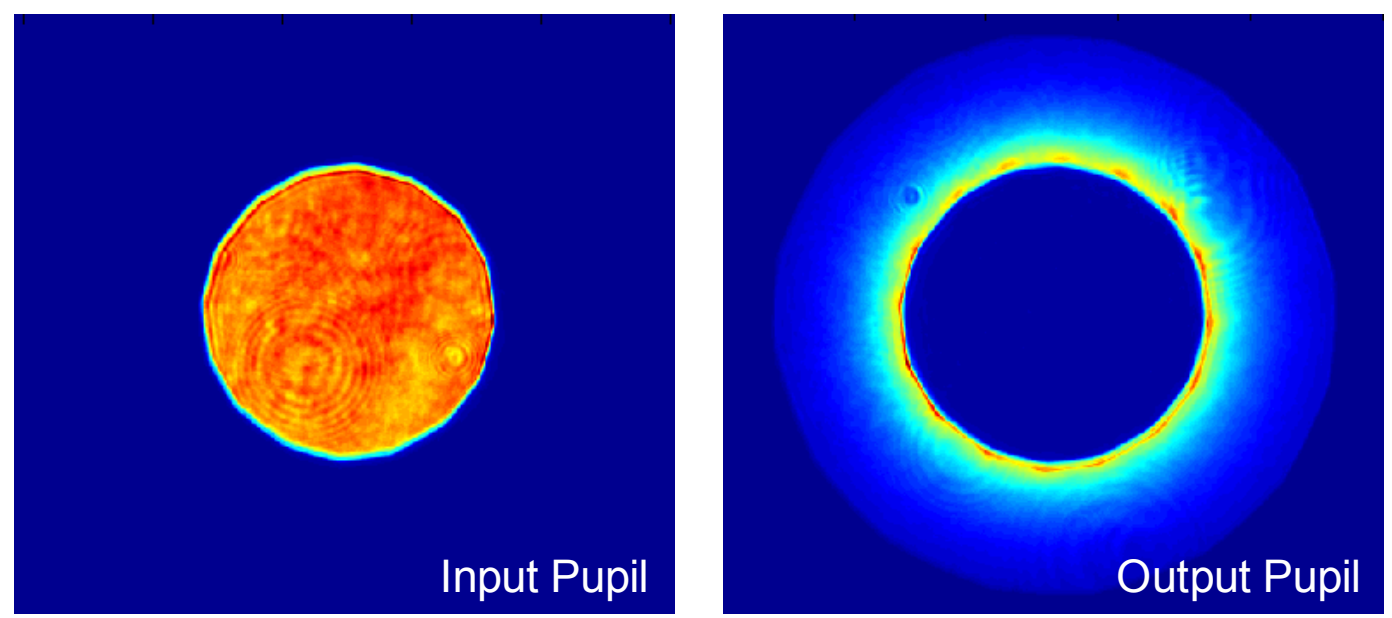

Figure 4. Input and output pupils for a clear input pupil used with a single stage vortex coronagraph configuration, as measured in the IRCT at $880 \mathrm{~nm}$, using a vortex phase mask manufactured by Beam Co. 
These preliminary experimental results thus provide a first-order verification of the basic physics of the dual-vortex approach, even in the presence of several limitations to our initial experiment, such as an input illumination that is not perfectly flat, potential errors in input pupil mask manufacture (such as a slightly off-axis central blockage), a central wavelength $(880 \mathrm{~nm})$ not well-matched to the vortex central wavelength $(800 \mathrm{~nm})$, broad bandwidth $(70 \mathrm{~nm})$, unblocked finite central confusion regions ${ }^{6}$ in our vortex masks, defocus in various planes, and stray light.
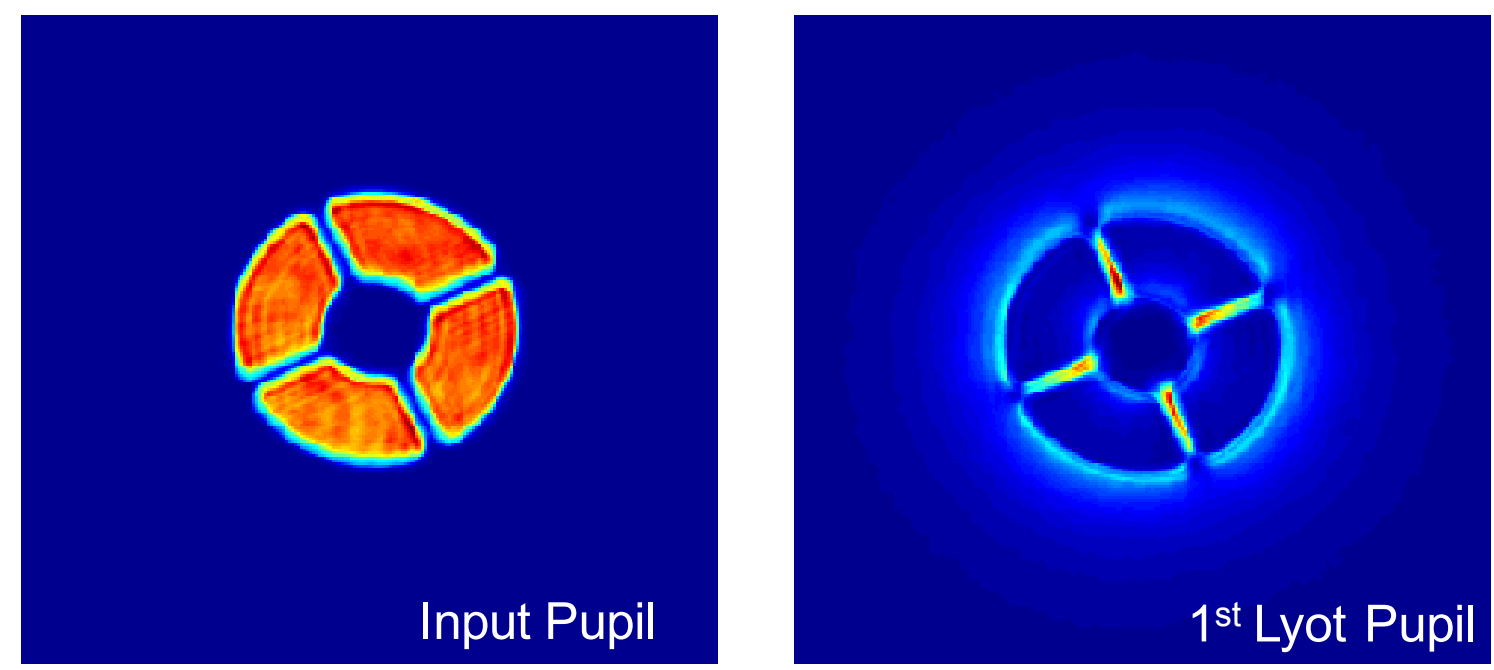

Figure 5. Input and output pupils for a single stage vortex configuration for a centrally blocked input pupil, as measured in the IRCT. The same vortex mask as in Figure 4 was used here.
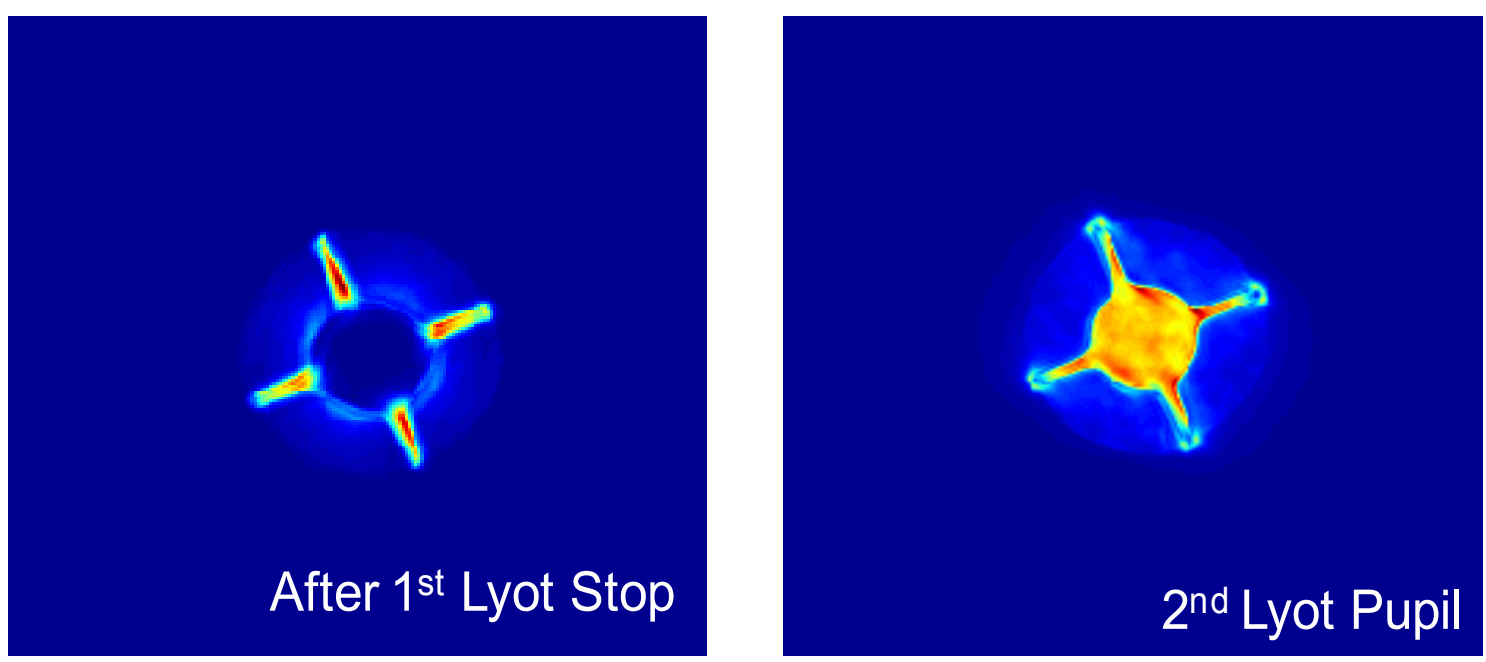

Figure 6. Left: apodized output pupil after the first vortex stage. This pupil distribution is the same as that of Fig. 5 (right hand side), but with the outer region cut off by an iris diaphragm. Right: the light distribution measured in the second Lyot plane of a double-vortex coronagraph by the IRCT, after a passage through a second second-order Beam Co. vortex phase mask. 


\section{THE EFFECT OF SECONDARY SUPPORTS}

One can also see in the final pupil image of Fig. 6 (right hand side) that the presence of secondary supports does not appear to be a significant limitation, as the residual light is well confined along the support legs, where it can also be blocked by the second Lyot stop. For further validation, Fig. 7 shows a numerical calculation of diffraction through a single-stage vortex coronagraph, assuming an input pupil with blockages due to both a central secondary mirror and secondary support legs. The same result is found here numerically: the scattered light is confined largely along the secondary supports, where it can be blocked. This "edge enhancement" property of vortex masks has been noted before . While the relative contributions of residual scattered light within the pupil due to the secondary and the support legs will of course depend on the size of the secondary and the widths of the legs, diffraction from the support legs can likely be held to tolerable levels by using narrow support legs and an oversized blocker in the second Lyot plane.
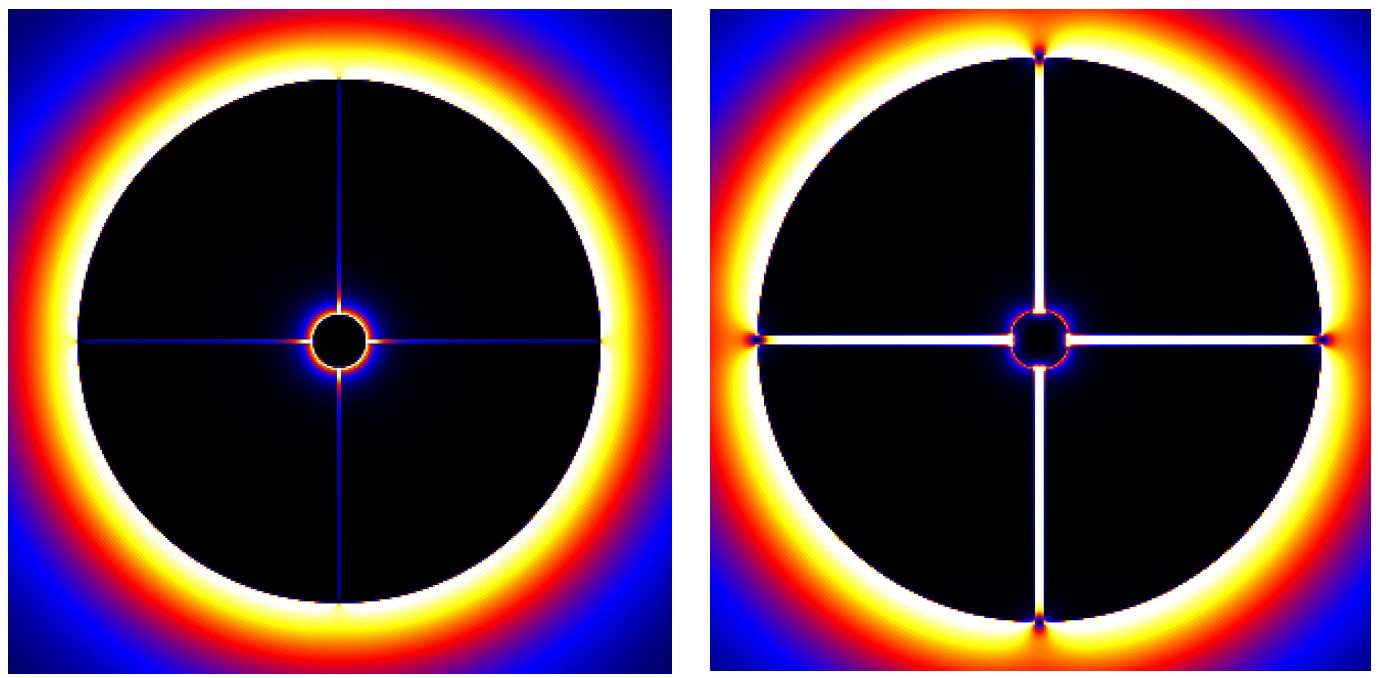

Figure 7. Calculation of the Lyot pupil downstream of a single vortex phase mask, assuming a secondary support spider with widths of $0.5 \%$ (left) and $2 \%$ (right) of the diameter of the primary. The residual light diffracted by the spider is confined largely along the secondary support legs.

\section{SPECKLE PHASE MEASUREMENTS WITH A DUAL-VORTEX CONFIGURATION}

On the other hand, if the central starlight beam is not blocked in the second Lyot plane of Fig. 6 (right), it can be put to good use. In particular, this starlight beam, which is spatially separated from the planet light in the outer pupil, can be used as a reference beam for interferometric measurements of wavefront errors ${ }^{3}$. To superpose the inner (starlight) reference beam with the outer beam (of starlight speckles and planet light), one simply needs to focus the second Lyot plane aperture with a common lens or mirror. This produces a common-mode interferometer configuration that can be used for phase measurements of focal plane speckles ${ }^{3}$ (Fig. 8). In detail, the narrow central starlight beam focuses to a broader focal plane distribution than the speckles arising from wavefront corrugations across the outer aperture. Thus the focused reference beam illuminates a wide field of speckles. By scanning the phase of the inner reference beam relative to the outer "science" beam, the phases of the focal plane speckles can thus be measured using the techniques of phaseshifting interferometry ${ }^{7}$ (Fig. 8). However, since the central reference beam power goes down as (d/D) ${ }^{2}$, while the residual starlight in the outer pupil goes down as $(\mathrm{d} / \mathrm{D})^{4}$, there are competing trends between reducing residual starlight in the larger pupil, and maximizing the reference power available in the inner pupil. 


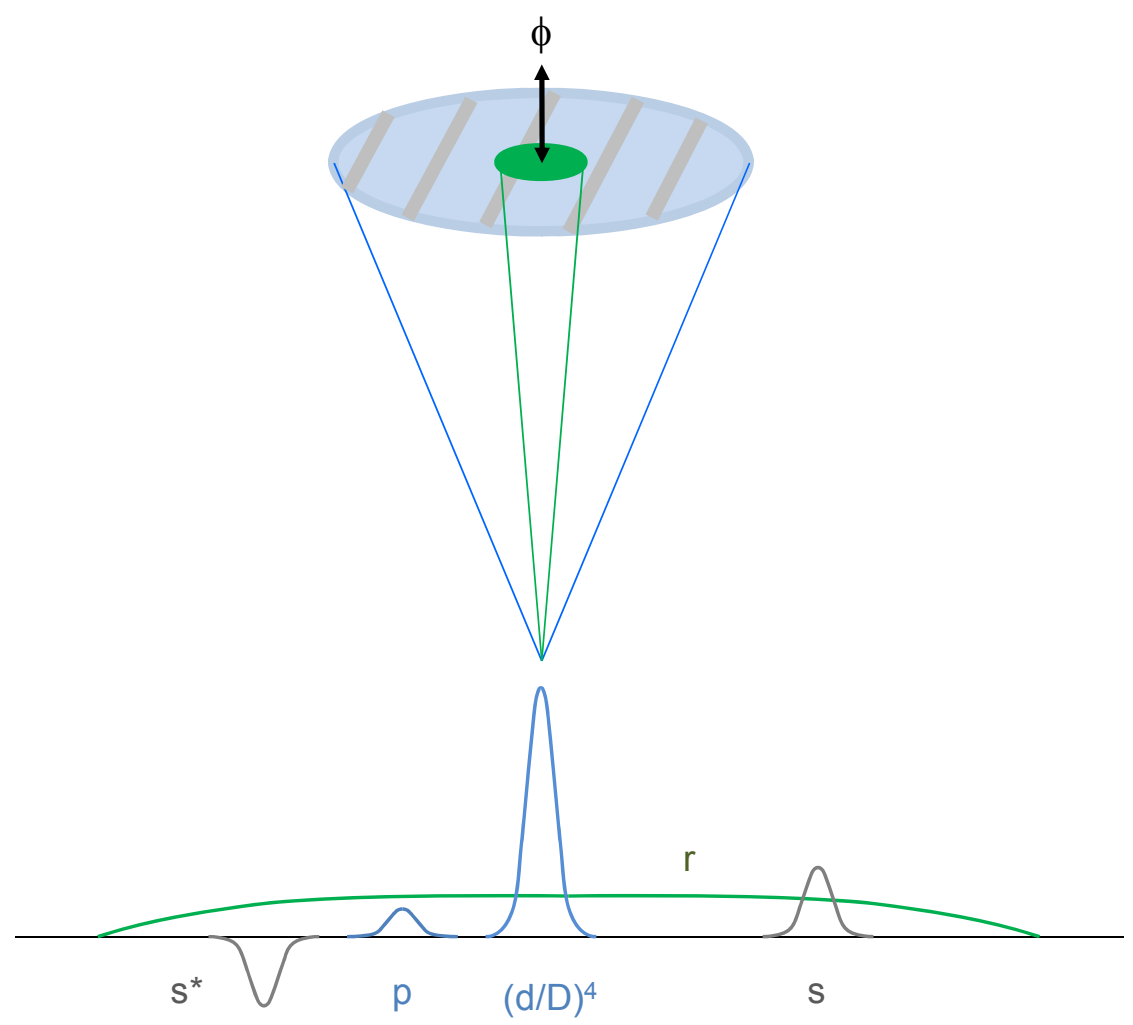

Figure 8. Direct focal-plane speckle-phase sensing is enabled by phase-shifting the central starlight beam produced by a dual-vortex configuration relative to the outer beam containing the scattered starlight (and planet light). The bottom part of the plot shows the broad reference point spread function ( $\mathrm{r}$ ) compared to the focused planet image $(\mathrm{p})$, the speckles $\left(\mathrm{s}, \mathrm{s}^{*}\right)$, and the residual starlight from the outer aperture (at the center of the image).

\section{RECENT LABORATORY MEASUREMENTS OF VORTEX REJECTION}

Finally, we briefly discuss recent laboratory measurements (Fig. 9) aimed at demonstrating deep and broadband starlight rejection, that we have carried out in JPL's High Contrast Imaging Testbed (HCIT) on a $4^{\text {th }}$ order vortex mask manufactured by JDS Uniphase. These experiments are discussed in more detail by Mawet et al. at this meeting, but here we present results from one final experimental run. In these experiments, the bandwidth was broadened by spectral polarization filtering ${ }^{4,8}$, implemented by including a polarizer and quarter-wave-plate upstream of the vortex to select the input circular polarization state, and a quarter-wave-plate and a polarizer downstream of the vortex to reject off-bandcenter leakage, which appears in the opposite circular polarization state ${ }^{8}$.

In the final experimental run described here, a dark hole was generated in the HCIT at a "central" wavelength, and the HCIT's deformable mirror was then left alone for further measurements, each of which were carried out with individual $2 \%$ bandwidth filters, so as to remove the effects of the source's spectral shape. Fig. 9 (right) shows two integrated contrast curves, the top one integrated over the entire dark hole (which ran from $2.75-6.3 \lambda / \mathrm{D}$ horizontally and to \pm 6.3 $\lambda / \mathrm{D}$ in the vertical direction), and the bottom curve for the top half of the dark hole, which showed lower scattered background light levels. The best result in an individual $2 \%$ filter was a (half-) dark hole contrast of $1.0 \times 10^{-8}$, and in a summed $10 \%$ passband, the corresponding best contrast was $1.6 \times 10^{-8}$. The full dark hole region was only of order a factor of two worse due to the slightly higher background levels. Note that even with a $20 \%$ band width, the rejection is $\approx 4 \times 10^{-8}$. Vortex phase masks have thus now demonstrated performance applicable to observations of, e.g., exozodiacal light and jovian exoplanets down to the $10^{-8}$ to $10^{-9}$ level, the latter assuming a factor of 10 or so improvement provided by speckle calibration procedures 9 . 

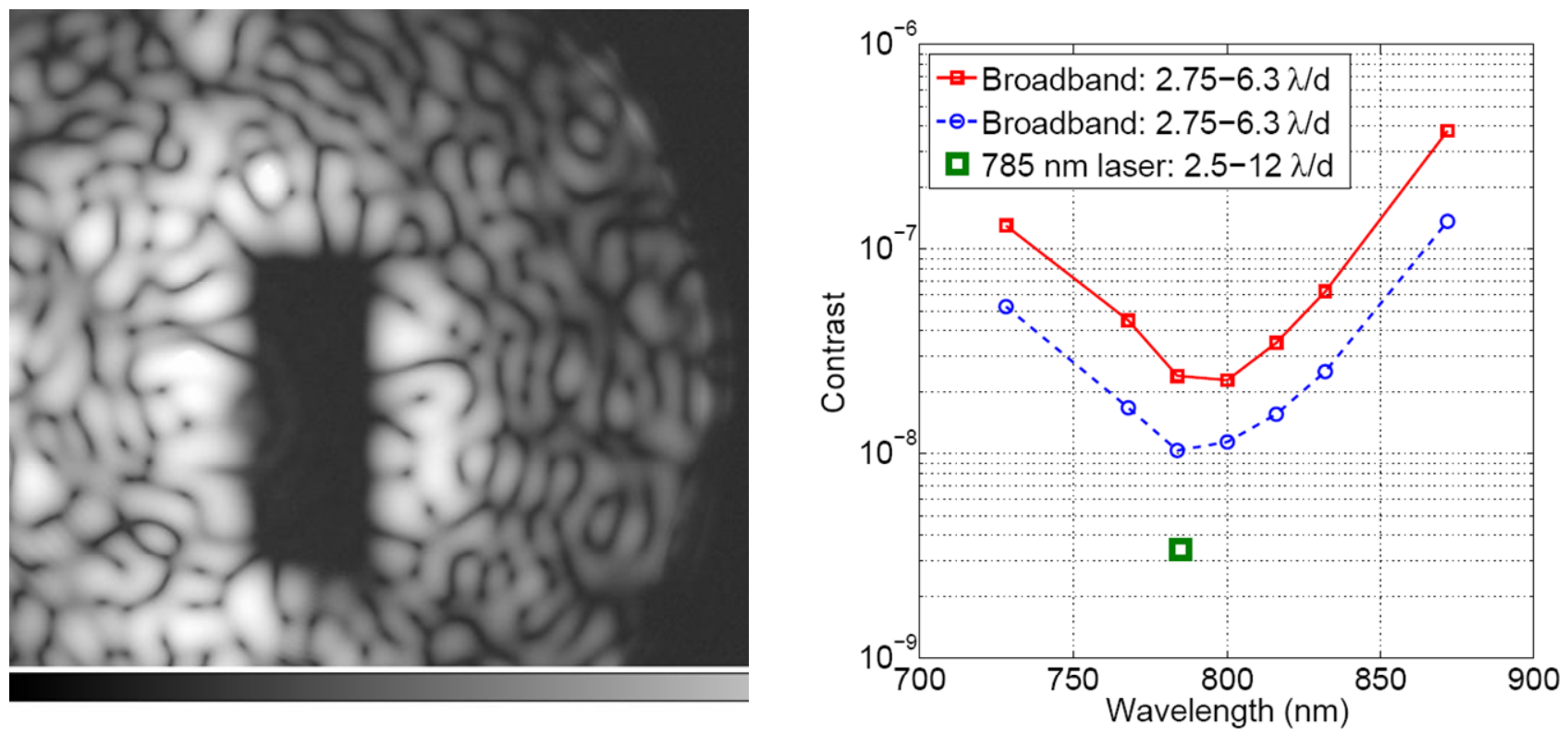

Figure 9. Left: An image-plane dark hole generated in the HCIT with a single-stage fourth-order vector vortex coronagraphic mask manufactured by JDS Uniphase. Right: Resultant broadband measurements of rejection levels. The top curve is for the full dark hole shown in the left-hand image, while the bottom curve is for the top half of the dark hole.

\section{SUMMARY}

The optical vortex coronagraph shows great potential in a number of areas, including its newly-discovered ability to operate behind an on-axis telescope, its ability to be configured to measure speckle phases directly, and its ability to operate over a broad band. As a result, the vortex coronagraph is well worth considering as a means to enable a coronagraphic space mission, and in particular, as a way to reduce aperture size and increase science yield. Indeed, given the small inner working angle of the vortex coronagraph, a significant science output should be possible with a telescope diameter of order $1-2 \mathrm{~m}$, thus potentially making feasible a small explorer class mission aimed at detecting exozodiacal light and jovian exoplanets.

This work was carried out at the Jet Propulsion Laboratory (JPL), California Institute of Technology (Caltech), under contract with the National Aeronautics and Space Administration (NASA).

\section{REFERENCES}

[1] Swartzlander, G.A. Jr., J. Opt. A 11, 094022 (2009)

[2] Mawet, D., Serabyn, G., Wallace, J. and Pueyo, L., Opt. Lett. 36, 1506-1508 (2011)

[3] Serabyn, E., Wallace, J.K. and Mawet, D., Applied Optics, in press (2011)

[4] Mawet, D., Pueyo, L., Moody, D., Krist, J. and Serabyn, E., Proc. SPIE 7739, 773914-1 (2010)

[5] Galicher, R., Baudoz, P. and Baudrand J., Astron. \& Astrophys. 530, A43 (2011)

[6] Mawet, D. , Serabyn, E., Liewer, K., Hanot, Ch., McEldowney, S., Shemo, D. and O’Brien, N., Opt. Exp. 17, $1902-$ 1918 (2009)

[7] Malacara, D. Optical Shop Testing, $2^{\text {nd }}$ ed., John Wiley \& Sons, Inc. (1992)

[8] Baba, N. and Murakami, N., Pub. Astron. Soc. Pac. 115,1363-1366 (2003)

[9] Lafreniere, D., Marois, C., Doyon, R., Nadeau, D. and Artigau, E., Astrophys. J. 660, 770-780 (2007) 